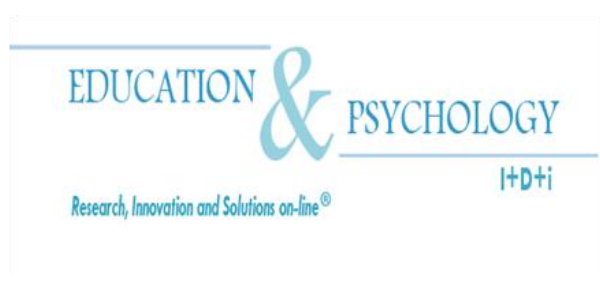

\title{
Cyberbullying en una muestra de estudiantes de Educación Secundaria: Variables moduladoras y redes sociales
}

\section{Benito León del Barco, Elena Felipe Castaño, Fernando Fajardo Bullón, Teresa Gómez Carroza}

${ }^{1}$ Departamento de Psicología y Antropología, Universidad de Extremadura

\section{España} 10071 Cáceres. España. E-mail: bleon@unex.es

(C) Education y Psychology I+D+i and Editorial EOS (Spain) 


\section{Resumen}

Introducción. Las formas tradicionales de maltrato entre iguales han cambiado con el transcurrir del tiempo, apareciendo manifestaciones más específicas que se sirven de las nuevas tecnologías de la información y de la comunicación. Esta nueva forma de maltrato se denomina cyberbullying. Con nuestro estudio pretendemos analizar la prevalencia del fenómeno $c y$ berbullying y de los diferentes tipos en la Comunidad de Extremadura y qué papel juegan el género, la edad y ser usuario de redes sociales.

Método. El número de participantes fue de 1700 estudiantes de Educación Secundaria de Extremadura, pertenecientes a 22 centros públicos y privados-concertados españoles. Hemos utilizado el instrumento denominado Cuestionario Cyberbullying.

Resultados .El porcentaje medio de agresores es de 6.4\% y el de víctimas 6\%. La variable sexo muestra una influencia significativa sobre la prevalencia de ser víctima con el móvil $\left(\chi^{2}=\right.$ 3.844; $\mathrm{gl}=1 ; \mathrm{p}<.048)$ y ser agresor utilizando internet $\left(\chi^{2}=3.947 ; \mathrm{gl}=1 ; \mathrm{p}<.047\right)$. Con relación al curso, sólo hemos encontrado una influencia significativa sobre la prevalencia de ser víctima con el móvil $\left(\chi{ }^{2}=14.955 ; \mathrm{gl}=3 ; \mathrm{p}<.002\right)$. Respecto a la influencia de la variable ¿Conoces redes sociales? en la prevalencia del fenómeno cyberbullying en la modalidad de internet perspectiva agresor, hemos encontrado correlación significativa entre ambas variables $\left(\chi^{2}=\right.$ 27.26; $\mathrm{gl}=3 ; \mathrm{p}<.000)$.

Discusión y Conclusiones. Nuestros datos están próximos a los obtenidos en la investigación del 2006, "Violencia Escolar: El maltrato entre iguales en la Educación Secundaria" del Defensor del Pueblo y se encuentran más alejados de otras investigaciones. Respecto a las variables que influyen en la prevalencia del fenómeno cyberbullying, nuestro estudio pone de manifiesto que las variables sexo, curso y redes sociales muestran una influencia significativa.

Palabras Clave: Cyberbullying, violencia, adolescencia, educación secundaria, uso de internet, redes sociales, medios de comunicación, alumnado. 


\title{
Cyberbullying in a Sample of Secondary Students: Modu- lating Variables and Social Networks
}

\begin{abstract}
Introduction. Traditional forms of bullying have changed over time, appearing more specific statements that use the new technologies of information and communication. This new form of abuse is called cyberbullying. In our study we analyze the prevalence of the phenomenon of bullying and cyberbullying different types in the Community of Extremadura and what role do gender, age and a user of social networks.
\end{abstract}

Methods. The number of participants was 1700 secondary school students from Extremadura, from 22 public and private-arranged. We used an instrument: Questionnaire Cyberbullying.

Results. The percentage of offenders is $6.4 \%$ and $6 \%$ of victims. The gender variable shows a significant influence on the prevalence of falling victim to the mobile $\left(\chi^{2}=3,844\right.$, df $=1, p$ $<.048)$ and being an aggressor using the internet $\left(\chi^{2}=3,947, \mathrm{df}=1, \mathrm{p}<.047\right)$. With regard to the course, we only observed a significant influence on the prevalence of falling victim to the mobile $\left(\chi^{2}=14,955, \mathrm{df}=3, \mathrm{p}<.002\right)$. Regarding the influence of social networks variable Do you know? in the prevalence of cyberbullying phenomenon in the form of internet aggressor perspective, we found significant correlation between both variables $\left(\chi^{2}=27.26\right.$, df $=3, p$ $<.000)$.

Discussion and Conclusion. Our data are close to those obtained in the investigation of 2006, "School Violence: Bullying in Education Secondary "Ombudsman and further away from other investigations. Regarding the variables that influence the prevalence of cyberbullying phenomenon, our study shows that variables sex, grade and social networks show a significant influence.

Keywords: Cyberbullying, violence, adolescence, secondary education, internet use, social networks, media, students.

Received: 03/08/12 Initial acceptance: 06/10/12 Final acceptance: 07/20/12 


\section{Introducción}

El maltrato o abuso entre iguales es una conducta de persecución física y/o psicológica que realiza el alumno o alumna contra otro, al que elige como víctima de repetidos ataques (Benítez y Justicia, 2006; Olweus, 1983). Actualmente, el auge exponencial en el uso de las nuevas tecnologías de la información y de la comunicación, así como el dominio y familiaridad de nuestro jóvenes con estas nuevas tecnologías (generación interactiva) ha provocado que las formas tradicionales de maltrato entre iguales cambien con el transcurrir del tiempo, apareciendo manifestaciones más específicas que se sirven de las nuevas tecnologías de la información y de la comunicación para acosar a la víctima. Esta nueva forma de maltrato se denomina cyberbullying (Ortega, Calmaestra y Mora-Merchan, 2008; Smith, Mahdavi, Carvalho y Tippet, 2006) y consistiría en una agresión intencional, por parte de un grupo o un individuo, utilizando recurrentemente formas electrónicas (móviles, internet) de contacto sobre una víctima que no puede defenderse por sí sola.

Este tipo de maltrato presenta aspectos comunes con las formas tradicionales de bullying, pero también unas características particulares que lo diferencian (Heirman y Walrave, 2009; Li, 2008; Ortega, Calmaestra y Mora-Merchan, 2008; Slonge y Smith, 2008; Ybarra y Mitchell, 2004) en el cyberbulling no existen lugares donde estar seguros lo que desarrolla mayor inseguridad en la víctima; el hecho de que el acoso pueda llegar incluso a tu propia casa provoca sentimientos de indefensión y desprotección. El acoso se hace público y puede ser observado indefinidamente por una mayoría de espectadores. La fuerza física o el tamaño no afecta, el acosador digital no tiene que ser más fuerte que sus víctimas y suele tener buenas relaciones con los profesores. Por último, existe un desconocimiento y anonimato de los agresores que provoca en las victimas sentimientos de impotencia.

¿Podemos clasificar el cyberbullying en diferentes tipos? Se puede utilizar para categorizarlo la vía por la que se produce el acoso (Smith, Mahdavi, Carvalho y Tippet, 2006) o la acción que se realiza (Willard, 2005). Incluso para algunos investigadores el cyberbullying no es más que un modo disimulado de acoso verbal y escrito (Mason, 2008). En general, se puede utilizar el móvil para enviar mensajes de texto amenazadores o mensajes multimedia como fotografías y videos de las víctimas y para realizar llamadas acosadoras, silenciosas, a horas inadecuadas, con alto contenido sexual...; se puede utilizar el correo electrónico para enviar 
mensajes insultantes, vejatorios y ofensivos; se puede utilizar la mensajería instantánea y páginas WEB para ridiculizar y difamar a la víctimas... En definitiva se puede acosar mediante el correo electrónico, el móvil, la mensajería instantánea y a través de páginas WEB.

Los primeros estudios sobre la incidencia del cyberbullying surgen en Estados Unidos (Finkelhor, Mitchell y Wolak, 2000; Ybarra y Mitchell, 2004), Reino Unido (Balding, 2005; Noret y Rivers, 2006; Smith, Mahdavi, Carvalho y Tippet, 2006), Cánada (Beran y Li, 2005) y Australia (Campbell, 2005). Más recientemente se han desarrollado investigaciones en Suecia, Holanda y Grecia (Kapatzia y Syngollitou, 2007; Slonje y Smith, 2008). Todos estos estudios constatan el problema del cyberbullying, oscilando los porcentajes de estudiantes que han sufrido episodios de acoso con las nuevas tecnologías del 5\% al 20\%. Nuevas investigaciones realizadas en los países pioneros como Estados Unidos manifiestan que el fenómeno va en aumento y, por tanto, se acrecienta el problema y las consecuencias del mismo, Raskauskas y Stoltz (2007) encuentran que cerca del $49 \%$ de los encuestados había sido víctima de cyberbullying y un $21 \%$ había agredido a otros.

En España, los primeros datos que tenemos de este fenómeno provienen del Informe "Violencia Escolar: El maltrato entre iguales en la Educación Secundaria" del Defensor del Pueblo (2006). En este estudio se observa que un 5,5\% de los estudiantes que se reconocen como víctimas, identifican las nuevas tecnologías como instrumento para los abusos que reciben. En el caso de los agresores la incidencia es del 4,8\%. Ortega, Calmaestra y MoraMerchan (2008) con una muestra de 830 estudiantes de ESO de la ciudad de Córdoba encuentran que un 26,6\% de los participantes están implicados directamente con el fenómeno cyberbullying. Un 10,8\% como víctimas y un 15,8\% como agresores y agresores victimizados. Destaca este estudio una mayor incidencia del cyberbullying a través de internet que el que se produce a través del móvil.

Jiménez y García (2010) con una muestra de 180 estudiantes de secundaria, utilizando el mismo cuestionario de Ortega, Calmaestra y Mora-Merchan (2008) observan que un 43,3\% de los participantes están inmersos en el problema, un 28,9\% como víctimas y un $14,4 \%$ como agresores. De las dos tecnologías analizadas, los participantes prefieren el acoso con internet frente al acoso con el móvil. Estévez, Villardón, Calvete, Padilla y Orue (2010) con una muestra de 1431 adolescentes de edades comprendidas entre 13 y 17 años, y con un cuestionario, el $C B Q-V$, desarrollado por las autoras para medir la victimización de cyberbullying, 
evidencian que el 30,1\% de los adolescentes declaran haber sufrido algún tipo de cyberagresión. Del Río, Sábada y Bringué (2010) han encuestado a 13.000 menores de entre 6 y 18 años mediante un cuestionario online. Entre sus resultados destacan que un 5\% de los menores entre 10 y 18 años han "utilizado internet para perjudicar a alguien", aunque parecen más propensos los chicos, $7 \%$ que las chicas, $4 \%$. Como víctimas un $8 \%$ declara que "alguien le ha perjudicado a través de internet", las chicas se reconocen más atacadas que los chicos, 9\% frente al $7 \%$.

¿Qué variables influyen en la prevalencia del cyberbullying? Las más estudiadas han sido la edad y el sexo, sin embargo los resultados de las diferentes investigaciones hasta el momento no son concluyentes (Li, 2006; Noret y Rivers, 2006; Slonje y Smith, 2008). En nuestro país, Ortega, Calmaestra y Mora-Merchan (2008) encuentran en su estudio que ninguna de las dos variables muestra una influencia significativa, aunque la variable sexo presenta cierta familiaridad con la prevalencia del bullying, las chicas suelen ser más víctimas de las conductas de acoso y los chicos más agresores. Por otro lado, Estévez, Villardón, Calvete, Padilla y Orue (2010) encuentran una mayor prevalencia en las chicas que entre los chicos. Para Del Río, Sábada y Bringué (2010) el sexo y la edad son variables vinculadas a una mayor propensión a ser víctima o verdugo en un escenario de cyberbullying. Estos mismos autores se plantean una cuestión interesante ¿están los usuarios de redes sociales (Tuenti, Facebo$o k . .$.$) más expuestos al fenómeno cyberbullying? Según sus resultados, ser usuario de redes$ sociales es una variable que incrementa sensiblemente la posibilidad de sufrir acoso a través de internet, los usuarios de internet que no tienen perfiles sociales, están menos expuestos a estas conductas.

\section{Objetivos e hipótesis}

Con este estudio pretendemos: 1. Conocer la prevalencia del fenómeno cyberbullying y de los diferentes tipos en la Comunidad de Extremadura y 2. Analizar el papel que juegan el género, la edad y ser usuario de redes sociales en la prevalencia de víctimas y agresores en el fenómeno cyberbullying.

Se han planteado las siguientes hipótesis: 1. Existirán asociaciones significativas entre la ocurrencia de ser víctima a través del móvil o internet con el género. 2. Existirán asociaciones significativas entre la ocurrencia de ser agresor utilizando el móvil o internet con el género. 3. Existirán correlaciones significativas entre ser víctima mediante el móvil o internet y la 
edad (medida indirectamente por curso). 4. Existirán correlaciones significativas entre ser agresor utilizando el móvil o internet y la edad (medida indirectamente por curso). 5. Existirán asociaciones significativas entre la incidencia de víctimas en el fenómeno cyberbullying y el conocimiento de redes sociales. 6. Existirán asociaciones significativas entre la incidencia de agresores en el fenómeno cyberbullying y el conocimiento de redes sociales.

\section{Método}

\section{Participantes}

La selección de los estudiantes se realizó mediante un muestreo polietápico, estratificado por conglomerados y selección aleatoria de los grupos en los centros que disponían de varias líneas en los cursos $1^{\circ}, 2^{\circ}, 3^{\circ}$ y $4^{\circ}$ de la ESO. Los estratos que se consideraron fueron los Centros de Recursos de Profesores (CPRs) que dividen en 18 zonas geográficas la Comunidad de Extremadura, cada CPR tiene asignados unos determinados Centros de Secundaria (IES e IESO). El muestreo por conglomerado consistió en seleccionar al azar dos centros de cada CPR. Algunos centros declinaron la participación en el estudio.

El número de participantes se determinó a partir del número de alumnos matriculados en secundaria en Extremadura durante el curso 2008-2009, considerando un error muestral de $3 \%$ y un nivel de confianza de 95,5\%. Finalmente, el número de participantes fue de 1700 estudiantes de Educación Secundaria de Extremadura, pertenecientes a 22 centros, 17 públicos y 5 privados-concertados. La distribución por género y nivel educativo de los participantes es la que podemos ver en la tabla 1 .

Tabla 1. Distribución del número de participantes según género y curso.

\begin{tabular}{lllll}
\hline & & Mujer & Varón & Total \\
\hline Curso de los alumnos & Primero de la ESO & 220 & 254 & 474 \\
\cline { 2 - 5 } & Segundo de la ESO & 214 & 235 & 452 \\
\cline { 2 - 5 } & Tercero de la ESO & 223 & 176 & 400 \\
\cline { 2 - 5 } & Cuarto de la ESO & 207 & 175 & 382 \\
\hline Total & & 864 & 840 & 1708 \\
\hline
\end{tabular}




\section{Instrumentos}

Hemos utilizado un instrumento: Cuestionario Cyberbullying (Ortega, Calmaestra y Mora Merchán, 2007). Este cuestionario según sus autores está basado en el de Smith, Mahdavi, Carvalho y Tippet (2006) al que se le han reducido de forma significativa el número de ítems. Las preguntas abarcan dos modalidades: situaciones de cyberbullying a través del teléfono móvil y situaciones en las que se utiliza Internet. Sobre el cuestionario original se han añadido preguntas que hacen referencia a las vías por las que se ejerce el acoso, a la comparación del cyberbullying con el maltrato tradicional, a los sentimientos que provocan las acciones de cyberbullying tanto en víctimas como agresores y a las estrategias de afrontamiento utilizadas. Hemos descrito los cuatro ítems que hacen referencia a ser víctima o agresor utilizando el móvil o internet en un formato Likert con cuatro intervalos en forma numérica de 1 al 4, que representan un continuo que va desde Ninguna a Varias veces a la semana. También, hemos añadido cuestiones relativas a la participación en redes sociales.

\section{Procedimiento}

Los datos que presentamos forman parte de una investigación más amplia relacionada con acoso escolar y el cyberbullying en centros de educación primaria y secundaria de Extremadura. El procedimiento seguido para la obtención de datos fue mediante la administración de los cuestionarios por grupo de clase. Previamente se había informado al centro y a los padres de los objetivos de la investigación y se había solicitado permiso al centro y los padres para su administración y a los participantes su consentimiento informado de forma verbal. Se aseguró a los participantes la confidencialidad de los datos obtenidos y su utilización exclusiva para fines de investigación. La administración de los cuestionarios ocupaba en torno a media hora y se llevó a cabo a lo largo de un mes durante el tiempo de tutorías.

\section{Análisis de datos}

Para analizar la incidencia de víctimas y agresores en el fenómeno cyberbullying se presentan los datos en términos de frecuencia relativas (porcentajes). Para estudiar la influencia que pudieran tener variables como el género, el curso y el uso de redes sociales en la prevalencia del cyberbullying se han utilizado pruebas Chi-cuadrado de independencia, estas pruebas no paramétricas se destinan al contraste de hipótesis (asociación entre una variable dependiente y otra independiente) cuando ambas variables son de tipo nominal. En esta investigación la variable dependiente ha sido la ocurrencia frente a la no ocurrencia de ser víctima o agresor a través del móvil o de internet. Las variables independientes han sido el género, 
curso y conocimiento de redes sociales. Por último, cuando se han encontrado asociaciones significativas para estimar la magnitud de la correlación entre las variables se ha utilizado el coeficiente Phi y la V de Cramer (Tablas de contingencia 2x2) y el Coeficiente de contingencia cuando al menos una de las dos variables tiene 3 o más categorías.

\section{Resultados}

En la Figura 1, se presentan los porcentajes de alumnos que utilizan el móvil, el ordenador, acceso a internet, redes sociales y conexión diaria a las redes sociales. Como interesante destacamos que un $90.4 \%$ tienen ordenador y el $81.5 \%$ se conecta diariamente a las redes sociales (Facebook y Tuenti)

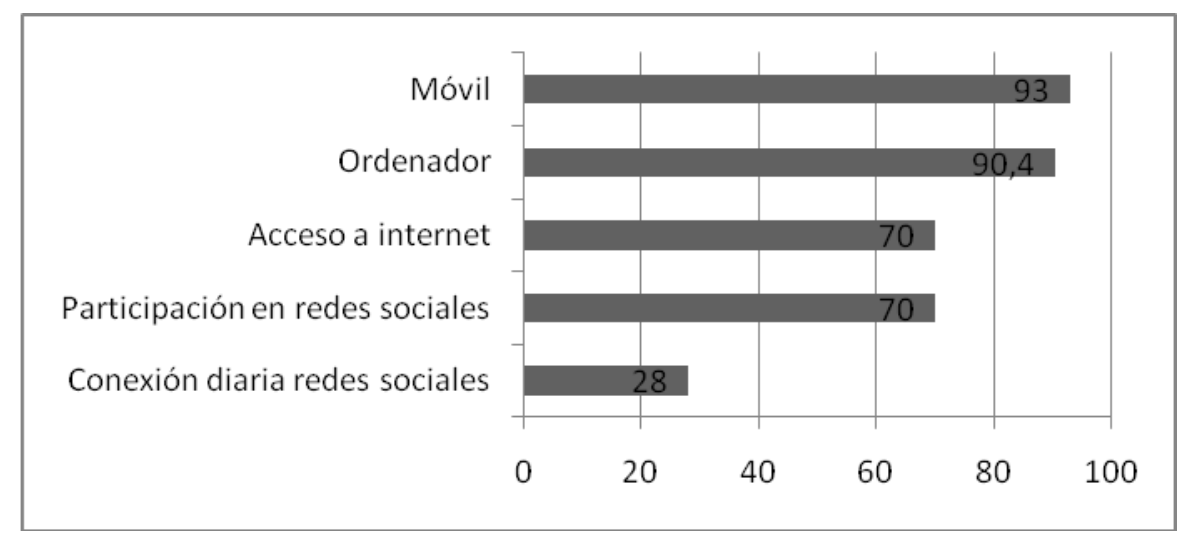

Figura 1. Porcentajes de alumnos usuarios de TICs

Con relación a ser agresor y víctima utilizando las nuevas tecnologías, el porcentaje medio de agresores es de $6.4 \%$ y el de víctimas $6 \%$. El porcentaje de agresores con el móvil es $5.5 \%$ y $6 \%$ el de víctimas. A través de internet el porcentaje de agresores sube al $7.3 \%$ y el de víctimas es de 6\%. En la tabla 2, podemos ver los porcentajes de los diferentes tipos de cyberbullying con el móvil y a través de internet. Con el móvil el porcentaje mayor se manifiesta mediante llamadas y con internet a través del Messenger.

Tabla 2. Porcentajes de víctimas en los diferentes tipos de Cyberbullying.

\begin{tabular}{lll}
\hline & Modalidades & Víctimas \\
\hline \multirow{2}{*}{ Móvil } & Mensajes MMS & $3.1 \%$ \\
\cline { 2 - 3 } & Llamadas & $22.7 \%$ \\
\cline { 2 - 3 } & SMS (Mensajes cortos) & $15.5 \%$ \\
\hline & E-mail & $10.2 \%$ \\
\cline { 2 - 3 } & Salas Chat & $11.2 \%$ \\
\hline
\end{tabular}




\begin{tabular}{lll}
\hline \multirow{2}{*}{ Internet } & Messenger & $25.5 \%$ \\
\cline { 2 - 3 } & Páginas WEB & $12.2 \%$ \\
\cline { 2 - 3 } & Redes sociales & $3.7 \%$ \\
\hline
\end{tabular}

Respecto a las variables que influyen en la prevalencia del fenómeno cyberbullying, hemos analizado el sexo, el curso y ser usuario de redes sociales. Con relación al sexo, hemos encontrado una influencia significativa sobre la prevalencia de ser víctima con el móvil $\left(\chi^{2}=\right.$ 3.844; $\mathrm{gl}=1 ; \mathrm{p}<.048)$ y ser agresor utilizando internet $\left(\chi^{2}=3.947 ; \mathrm{gl}=1 ; \mathrm{p}<.047\right)$. En la tabla 3 podemos observar que la relación significativa se establece entre ser mujer y ser víctima con el móvil y ser agresor con internet (Residuos corregidos > +1.9). Probablemente, las chicas están más involucradas que los chicos en la dinámica cyberbullying, como víctimas y agresoras. Sin embargo, hemos comprobado el tamaño del efecto a través de los índices de asociación Phi y V de Cramer, que nos dan un valor de .100, por lo que podemos afirmar que la relación encontrada es débil. No se han encontrado asociaciones significativas con la prevalencia agresor con el móvil $\left(\chi^{2}=3.751 ; \mathrm{gl}=1 ; \mathrm{p}<.053\right)$ y víctima con internet $\left(\chi^{2}=1.217\right.$; $\mathrm{gl}=1 ; \mathrm{p}<.270)$.

Tabla 3. Tabla de contingencia ( $2 \times 2)$, cyberbullying*sexo y resultados Prueba Chi-Cuadrado.

\begin{tabular}{|c|c|c|c|c|c|c|c|c|}
\hline & & Sexo & Recuento & $\%$ & $\begin{array}{l}\text { Residuos } \\
\text { Corregidos }\end{array}$ & $\chi^{2}$ & $\mathrm{gl}$ & $\begin{array}{c}\text { Sig. } \\
\text { (bilateral) }\end{array}$ \\
\hline \multirow{4}{*}{$\begin{array}{c}\text { Víctima a través } \\
\text { del móvil }\end{array}$} & \multirow[b]{2}{*}{ NO } & Mujer & 790 & $93.1 \%$ & -2 & \multirow{4}{*}{3.844} & \multirow{4}{*}{1} & \multirow{4}{*}{.048} \\
\hline & & Varón & 790 & $95.3 \%$ & 2 & & & \\
\hline & \multirow[b]{2}{*}{ SI } & Mujer & 59 & $6.9 \%$ & 2 & & & \\
\hline & & Varón & 39 & $4.7 \%$ & -2 & & & \\
\hline \multirow{4}{*}{$\begin{array}{l}\text { Agresor con } \\
\text { internet }\end{array}$} & \multirow[b]{2}{*}{ NO } & Mujer & 783 & $91.4 \%$ & -2 & \multirow{4}{*}{3.947} & \multirow{4}{*}{1} & \multirow{4}{*}{.047} \\
\hline & & Varón & 784 & $93.9 \%$ & 2 & & & \\
\hline & \multirow[b]{2}{*}{ SI } & Mujer & 74 & $8.6 \%$ & 2 & & & \\
\hline & & Varón & 51 & $6.1 \%$ & -2 & & & \\
\hline
\end{tabular}

Con relación al curso, sólo hemos encontrado una influencia significativa sobre la prevalencia de ser víctima con el móvil $\left(\chi^{2}=14.955 ; \mathrm{gl}=3 ; \mathrm{p}<.002\right)$. En la tabla 4 podemos observar que la relación significativa se establece entre los alumnos de $1^{\circ}$ de la ESO y ser víctima con el móvil (Residuos corregidos > +1.9). La intensidad de la relación encontrada es débil (Coeficiente de contingencia $=.127$ ). Podemos afirmar que existe una tendencia a que 
los alumnos más jóvenes ( $1^{\circ}$ de la ESO) sean más víctimas con el móvil que los alumnos de los últimos cursos de la ESO.

Tabla 4. Tabla de contingencia (4x2), cyberbullying*curso y resultados Prueba Chi-Cuadrado.

\begin{tabular}{|c|c|c|c|c|c|c|c|c|}
\hline & & Cursos & Recuento & $\%$ & $\begin{array}{c}\text { Residuos } \\
\text { Corregidos }\end{array}$ & $\chi^{2}$ & gl & $\begin{array}{c}\text { Sig. } \\
\text { (bilateral) }\end{array}$ \\
\hline \multirow{8}{*}{$\begin{array}{l}\text { Víctima a través } \\
\text { del móvil }\end{array}$} & \multirow{4}{*}{ NO } & $1^{\circ} \mathrm{ESO}$ & 423 & $91.2 \%$ & -3.2 & \multirow{8}{*}{14.955} & \multirow{8}{*}{3} & \multirow{8}{*}{.002} \\
\hline & & $2^{\circ} \mathrm{ESO}$ & 411 & $94.1 \%$ & -0.1 & & & \\
\hline & & $3^{\circ} \mathrm{ESO}$ & 375 & $94.7 \%$ & 0.5 & & & \\
\hline & & $4^{a} \mathrm{ESO}$ & 371 & $97.4 \%$ & 3.0 & & & \\
\hline & \multirow{4}{*}{ SI } & $1^{\circ} \mathrm{ESO}$ & 41 & $8.8 \%$ & 3.2 & & & \\
\hline & & $2^{\circ} \mathrm{ESO}$ & 26 & $5.9 \%$ & 0.1 & & & \\
\hline & & $3^{\circ} \mathrm{ESO}$ & 21 & $5.3 \%$ & -0.5 & & & \\
\hline & & $4^{\mathrm{a}} \mathrm{ESO}$ & 10 & $2.6 \%$ & -3.0 & & & \\
\hline
\end{tabular}

Con relación a la influencia de la variable ¿Conoces redes sociales? en la prevalencia del fenómeno cyberbullying en la modalidad de internet perspectiva agresor, hemos encontrado correlación significativa entre ambas variables $\left(\chi^{2}=27.26 ; \mathrm{gl}=3 ; \mathrm{p}<.000\right)$. En la tabla 5 podemos observar que la relación se establece entre los que conocen las redes sociales y agreden sólo una o dos veces (Residuos corregidos > +1.9). La intensidad de la correlación encontrada fue moderada-baja (Coeficiente de contingencia $=.127$ ).

Tabla 5. Tabla de contingencia $(4 \times 2)$, agresor a través de internet* conoces redes sociales.

\begin{tabular}{|c|c|c|c|}
\hline \multicolumn{2}{|c|}{$\begin{array}{l}\text { ¿Cuántas veces te has metido con alguien } \\
\text { a través de internet? }\end{array}$} & \multicolumn{2}{|c|}{$\begin{array}{c}\text { ¿Conoces redes sociales: Tuenti, } \\
\text { Facebook...? }\end{array}$} \\
\hline & & $\mathrm{NO}$ & SI \\
\hline \multirow[t]{3}{*}{ Ninguna } & Recuento & 188 & 1366 \\
\hline & $\%$ Conoces redes sociales & $95.4 \%$ & $92.7 \%$ \\
\hline & Residuos corregidos & 1.4 & -1.4 \\
\hline \multirow[t]{3}{*}{ Sólo una o dos veces } & Recuento & 1 & 81 \\
\hline & $\%$ Conoces redes sociales & $.5 \%$ & $5.5 \%$ \\
\hline & Residuos corregidos & $-3,0$ & 3.0 \\
\hline \multirow[t]{3}{*}{ Una vez a la semana } & Recuento & 7 & 8 \\
\hline & $\%$ Conoces redes sociales & $3.6 \%$ & $.5 \%$ \\
\hline & Residuos corregidos & 4.2 & -4.2 \\
\hline \multirow[t]{3}{*}{ Varias veces a la semana } & Recuento & 1 & 18 \\
\hline & $\%$ Conoces redes sociales & $.5 \%$ & $12 \%$ \\
\hline & Residuos corregidos & -.9 & .9 \\
\hline
\end{tabular}

No hemos encontrado correlaciones entre las variables conocimiento de redes sociales y ser víctima a través de internet. Por último, considerando como variable los días a la semana 
de conexión a las redes sociales, hemos encontrado correlaciones significativas con la agresión a través de internet, aunque la intensidad es baja (Índice Pearson= .112; p<.000).

\section{Discusión y Conclusiones}

Con este trabajo pretendíamos analizar la prevalencia del Cyberbullying en una muestra de alumnos de Educación Secundaria. También, se han analizado la influencia del género, del curso y del acceso a las redes sociales en el fenómeno cyberbullying. Nuestros resultados manifiestan que, aproximadamente, el porcentaje medio de agresores es de $6.4 \%$ y el de víctimas $6 \%$, estos datos están próximos a los obtenidos en la investigación "Violencia Escolar: El maltrato entre iguales en la Educación Secundaria” del Defensor del Pueblo en España (2006) y se encuentran más alejados del $10.8 \%$ como víctimas y un $15.8 \%$ como agresores y agresores victimizados del estudio de Ortega, Calmaestra y Mora-Merchan (2008) y de la investigación de Buelga, Cava y Musitu (2010) que encuentran un 25\% de adolescentes acosados durante el último año por el teléfono móvil y un $30 \%$ a través de internet. Como comentábamos en la introducción, los primeros estudios internacionales encuentran unos porcentajes que oscilan entre el $5 \%$ y el $20 \%$.

¿Por qué estas diferencias en la incidencia del Cyberbullying en España? Estas diferencias tendrían varias causas, las diferencias existentes entre las distintas comunidades autónomas en cuanto a la generalización del uso de las TIC, el momento en qué se realiza el estudio y por último, la metodología. Con relación a la generalización del uso de las TIC, según la Encuesta sobre Equipamiento y Uso de las Tecnologías de Información y Comunicación en los Hogares, realizada por el Instituto Nacional de Estadística el año 2010, las comunidades autónomas que se encuentran por encima de la media española en el uso de ordenadores e internet son Aragón, Islas Baleares, Cataluña, Comunidad de Madrid, Comunidad Foral de Navarra y País Vasco. Cantabria y Comunidad Valenciana superan la media nacional en el indicador referido al uso del ordenador en los últimos tres meses. Por su parte, Extremadura se sitúa por debajo del umbral del $90 \%$ en el uso de Internet y Andalucía y Región de Murcia no superan el mencionado umbral en el caso de usuarios frecuentes de Internet. Con estos datos, por ejemplo, es fácil comprender la diferencia encontrada en la prevalencia del Cyberbullying entre nuestro estudio realizado en Extremadura y la investigación de Buelga, Cava y Musitu (2010) realizada en la Comunidad Valenciana. 
Respecto al momento en qué se realiza el estudio, según la Encuesta sobre Equipamiento y Uso de las Tecnologías de Información y Comunicación en los Hogares, el número de internautas creció un 7.1\% en el año 2010. El porcentaje de hogares que disponía de ordenador en el año 2007 era del 60.4\% frente a un 69\% del año 2010. El porcentaje de teléfono móvil ha pasado del 90\% al 95\%. En una sociedad sujeta a continuos cambios y a un aumento exponencial en el uso de las nuevas tecnologías de la información, los datos obtenidos por investigaciones realizadas en diferentes años son difíciles de integrar.

Pensamos que la comparación de las investigaciones resulta difícil por las diferentes metodologías utilizadas, especialmente consideramos factores relevantes en la metodología, la determinación del número de participantes y su representatividad y los instrumentos aplicados. Así, por ejemplo, el estudio pionero de Ortega, Calmaestra y Mora-Merchan (2008) se limita a la ciudad de Córdoba, no teniendo en cuenta zonas rurales y el estudio reciente de Buelga, Cava y Musitu (2010) utiliza un instrumento diferente al resto de trabajos. Por ahora, la mayoría de estos trabajos científicos por motivos educativos y sociales se limitan a analizar la prevalencia en determinadas zonas o comunidades, siendo unos más potentes que otros desde el punto de vista de la selección y determinación de la muestra, lo que dificulta la generalización de resultados. Sería necesario en un futuro que los instrumentos tuviesen una métrica común que facilitara la interpretación y la integración de resultados.

Respecto a las variables que influyen en la prevalencia del fenómeno cyberbullying, nuestro estudio pone de manifiesto que la variable sexo muestra cierta influencia, coincidiendo con Ortega, Calmaestra y Mora-Merchan (2008) que encuentran diferencias significativas entre chicos y chicas cuando consideran el cyberbullying a través del móvil, existiendo una mayor incidencia de víctimas en las chicas. De hecho, según la Encuesta sobre Equipamiento y Uso de las Tecnologías de Información y Comunicación en los Hogares, realizada por el Instituto Nacional de Estadística en el año 2008, disponían de móvil un 70\% de las niñas frente a un $62 \%$ de los niños. Otros estudios en nuestro país encuentran que las chicas son más victimizadas que los chicos (Buelga, Cava y Musitu, 2010; Estévez, Villardón, Calvete, Padilla y Orue, 2010). En general, podemos concluir que las diferencias entre chicos y chicas en la prevalencia del fenómeno cyberbullying no están claras o apuntan en direcciones diferentes (Li, 2006; Noret y Rivers, 2006; Mora-Merchan, 2008; Slonge y Smith, 2008), no obstante interpretar estas diferencias implica analizar la disposición del móvil y de internet entre los participantes. 
Con relación a la variable curso, los alumnos de primero de la ESO tienden a ser más victimizados que los alumnos de los últimos cursos a través del móvil, pero no con internet. Esta tendencia sucede también con el maltrato escolar, los malos tratos entre iguales son más frecuentes en la adolescencia temprana en comparación con etapas posteriores (Defensor del Pueblo, 2006; León, Felipe, Gómez y López, 2011; Sánchez y Cerezo, 2010). ¿Por qué esta tendencia se da en nuestros participantes en la modalidad de acoso con móvil y no con internet? Sólo un $65.8 \%$ de alumnos de primero de la ESO y un $67 \%$ de $2^{\circ}$ de la ESO tienen acceso a internet, frente a un $70.3 \%$ y $75.4 \%$ de alumnos de $2^{\circ}$ y $3^{\circ}$ de la ESO, respecto al porcentaje de alumnos que disponen de móvil son muy similares en todos los cursos de la ESO, prácticamente un $92 \%$.

Por otro lado, considerando que los malos tratos entre iguales son más frecuentes en los primeros niveles educativos de la ESO, y además según el Informe "Violencia Escolar: El maltrato entre iguales en la Educación Secundaria" del Defensor del Pueblo (2006) esta pauta sería, especialmente, relevante en el acoso verbal (me insultan y me ponen motes) y que estos alumnos de primer curso disponen en mayor porcentaje de la herramienta móvil (92\%) y menos internet (65.8\%), tendrían sentido los resultados encontrados. Sin duda, nuestros datos no coincidirían con aquellas comunidades donde la disposición del móvil y de internet sea diferente a la nuestra.

En general los alumnos de los primeros niveles de la ESO están más involucrados en los malos tratos y acosaran con las herramientas (móvil o internet) que tengan más en sus manos, sobre todo cuando se trata de un acoso verbal destinado a provocar daño en el círculo de amistades de otra persona o en la percepción de pertenencia al grupo (maltrato relacional). Para algunos investigadores el cyberbullying no es más que un modo disimulado de acoso verbal y escrito (Mason, 2008), habría que analizar en líneas futuras de investigación la relación entre las experiencias cyberbullying y las formas tradicionales de maltrato escolar. Para Li (2006) existiría una continuidad en el rol de víctima entre los episodios del acoso escolar y los del cyberbullying.

Como sabemos el acceso a las redes sociales ha aumentado vertiginosamente entre toda la población y, sobre todo, entre los jóvenes por el atractivo que les supone disponer de un medio virtual donde puedan participar de forma activa, íntima y relacionarse con los amigos. 
Con respecto al papel que pueda ejercer en la prevalencia del cyberbullying el acceso a redes sociales, nuestros resultados manifiestan una tendencia a la existencia de correlaciones entre el acceso a redes sociales (Conocimiento de las mismas y días de conexión a la semana) y la agresión a través de internet. Pocos claras están las relaciones entre acceso a redes sociales y ser víctima a través de internet. En definitiva, y coincidiendo con Del Río, Sábada y Bringué (2010), ser usuario de redes sociales es una variable que incrementa sensiblemente la posibilidad de acosar a través de internet.

La generalización de las TICs entre nuestros jóvenes nos enfrenta a un nuevo fenómeno que no sólo se concreta en el cyberbullying, sino también en otros riesgos online como el grooming (engatusamiento de un adulto para ganarse la confianza de un menor con fines de satisfacción sexual) o el sexting (envío de contenidos de tipo sexual por medio del teléfono móvil o internet). Las consecuencias del acoso en las víctimas, la preocupación social y la repercusión mediática de estos riesgos nos obliga a la adopción de iniciativas para afrontar el cyberbullying. Actualmente, aunque no hay medidas globales, se han promovido iniciativas de diversa naturaleza desde el gobierno español (proyecto "TIC-tac TIC tac" de Ministerio de Industria) y desde las diferentes autonomías. Entre las iniciativas de las organizaciones no gubernamentales destacamos el programa "Pantallas amigas" que surge con la finalidad de dotar a los menores de las herramientas adecuadas para un uso seguro y saludable de internet.

La escuela es la principal oportunidad que tiene nuestra sociedad para crear espacios de convivencia y cambios de actitudes, y los programas para concienciar y afrontar el cyberbullying deberían estar relacionados con los proyectos de convivencia escolar que se desarrollan en todos los centros. Es importante, también, dotar de recursos al profesorado para prevenir y tratar las diferentes modalidades del acoso (Álvarez-García, Rodríguez, GonzálezCastro, Núñez y Álvarez, 2010). El cyberbullying es un fenómeno que transciende el contexto escolar, buena parte del mismo tiene lugar en el propio hogar, y no podemos olvidarnos del papel de la familia en su prevención. 


\section{Referencias}

Álvarez-García, D., Rodríguez, C., González-Castro, P., Núñez, J.C. y Álvarez, L. (2010). La formación de los futuros docentes frente a la violencia escolar. Revista de Psicodidáctica, 15(1), 35-56.

Balding, J. (2005). Young People in 2004: The health-related behaviour questionnaire results for 40,430 young people between the ages of 10 and 15. Schools Health Education Unit: Exeter.

Benítez, J.L. y Justicia, F. (2006). El maltrato entre iguales: descripción del fenómeno. Electronic Journal of Research in Educational Psychology, 9 (4), 151-170.

Beran, T. y Li, Q. (2005). Cyber-harassment: a new method for an old behavior. Journal of Educational Computing Research, 3, 265-277.

Buelga, S., Cava, M.J. y Musitu, G. (2010). Cyberbullying: victimización entre adolescentes a través del teléfono móvil y de Internet. Psicothema, 22, 784-789.

Campbell, M. (2005). Cyberbullying: an old problem in a new guise? Australian Journal of Guidance and Couselling, 15, 68-76.

Defensor del Pueblo (2006). Violencia escolar: el maltrato entre iguales en la Educación Secundaria Obligatoria (1999-2006). Madrid: Publicaciones del Defensor del Pueblo.

Del Río, J., Sádaba, C. y Bringué, X. (2010). Menores y redes ¿sociales?: de la amistad al cyberbullying. Revista de Estudios de la Juventud, 88, 115-129.

Estévez, A., Villardón, L., Calvete, E., Padilla, P. y Orue, I. (2010). Adolescentes víctimas de cyberbullying: prevalencia y características. Revista de Psicología Clínica y de la Salud, 18, 73-89.

Finkelhor, D., Mitchell, K.J. y Wolak, J. (2000). Online victization: a report on the nation's youth. Alexandria, VA: National Center for Missing and Exploited Children.

Heirman, W. y Walrave, M. (2009). Asseing issues and concerns about the mediation of technology in Cyberbullying. Trípodos Extra, 1, 317-329.

Instituto Nacional de Estadística (2008). Encuesta sobre Equipamiento y Uso de las Tecnologías de Información y Comunicación en los Hogares. Recuperado el 10 Octubre de 2010, de http://www.ine.es/prensa/np517.pdf

Instituto Nacional de Estadística (2010). Encuesta sobre Equipamiento y Uso de las Tecnologías de Información y Comunicación en los Hogares. Recuperado el 10 Octubre de 2010, de http://www.ine.es/prensa/np620.pdf. 
Jiménez, A. y García, A. (2010). Cyberbullying: un estudio descriptivo. En J. J. Gázquez y M.C. Pérez (Eds.), Investigación en convivencia escolar: variables relacionadas (pp. 137-141). Granada: GEU.

Kapatzia, A. y Syngollitou, E. (2007). Cyberbullying in middle and high schools: prevalence, gender and age differences. Unpublished manuscript based on MSc Thesis of A. Kaptazia, University of Thessaloniki.

León, B., Felipe, E., Gómez, T. y López, V. (2011). Acoso escolar en la Comunidad de Extremadura vs. Informe español del Defensor del Pueblo (2006). Electronic Journal of Research in Educational Psychology, 9 (2), 565-586.

Li, Q. (2006). Cyberbullying in schools: A research of gender differences. School Psychology International, 27, 157-170.

Li, Q. (2008). A cros-cultural comparison of adolescents experience related to cyberbullying. Educational Research, 50 (3), 223-234.

Mason, K.L. (2008). Cyberbullying: a preliminary assessment for school personnel. Psychology in the School, 45, 323-348.

Mora-Merchan, J. (2008). Cyberbullying: un nuevo reto para la convivencia en nuestras escuelas. Informació Piscológica, 94, 60-70.

Noret, N. y Rivers, I. (2006). The prevalence of bullying by text message or email: results of a four year study. Póster presentado a British Psychological Society Annual Conference, Cardiff.

Olweus, D (1983). Low school achievement and agressive behaviour in adolescent boys. En D. Magnusson \& V. Allen (Eds.), Human developrnent. An interactional perspective (pp. 353-365). Nueva York: Academic Press.

Ortega, R., Calmaestra, J. y Mora, J. (2007). Cuestionario Cyberbullying. Universidad de Córdoba, instrumento no publicado.

Ortega, R., Calmaestra, J. y Mora, J. (2008) Cyberbullying. Internacional Journal of Psychology and Psychological Therapy, 8 (2), 183-192.

Raskauskas, J. y Stoltz, A. D. (2007). Involvement in traditional and electronic bullying among adolescents. Developmental Psychology, 43, 564-575.

Sánchez, C. y Cerezo, F. (2010). Variables personales y sociales relacionadas con la dinámica bullying en escolares de Educación Primaria. Electronic Journal of Research in Educational Psychology, 8 (3), 1015-1032.

Slonge, R. y Smith, P. (2008). Cyberbullying: another main type of bullying. Scandinavian Journal of Psychology, 49, 147-154. 
Smith, P.K., Mahdavi, J., Carvalho, C. y Tippett, N. (2006). An investigation into cyberbullying, its forms, awareness and impact, and the relationship between age and gender in cyberbullying. London: Anti-Bullying Alliance.

Willard, N. (2005). Educator's guide to cyberbullying and cyberthreats. Recuperado el 10 Octubre de 2010, de http.//new.csriu.org/cyberbully/docs/cbcteducator.pdf

Ybarra, M. y Mitchell, K. (2004). Youth engaging in online harassment: Associations with caregiver chid relationships, internet use and personal characteristics. Journal of Adolescence, 2, 319-336. 